[INVITED ARTICLE]

\title{
MERGE: BIOLINGUISTIC CONSIDERATIONS
}

\author{
CEDRIC BOECKX \\ ICREA/Universitat de Barcelona
}

\begin{abstract}
This paper examines the nature of the operation Merge, the recursive procedure at the heart of the human language faculty. The central claim of this paper is that in order to reflect on the brain basis of Merge, the operation should be formulated in as much a generic way as possible. To achieve this syntactic combination must be dissociated from lexical influence. I argue that syntactic computation boils down to a rhythm: an interleaving of Merge and Spell Out applications, that may be understandable in terms of brain-level oscillations.*
\end{abstract}

Keywords: Biolinguistics, Merge, Oscillation, Phase, Spell-Out

\section{Biolinguistic Concerns}

In this essay I want to reflect on the biological foundations of our seemingly unique cognitive ability to construct 'finite-yet-unbounded' systems, in the sense of systems yielding (generating, licensing) endlessly many complex expressions in various realms such as language, mathematics, music, and morality. That a recursive procedure - perhaps a different one for each

* Portions of this material were presented at the 30th meeting of the English Linguistic Society of Japan hosted by Keio University, as part of the workshop on 'Basic Operations of Syntax.' This paper also draws on my presentation "Not Chomskyan Enough" at the Symposium on 'Language, Cognition, and Human Nature: Prospects of Linguistics' that took place at the same meeting. I am grateful to the Society for inviting me to deliver these talks, and to the audience for useful comments and reactions. I'd like to dedicate this work to the memory of the late Jean-Roger Vergnaud (1945-2011), who was a good friend, and mentor, and who I think was on to something very important- 'much more' indeed - when he wrote "On a certain notion of "occurrence": The source of metrical structure and of much more" (Vergnaud (2003)). The present work was made possible through a Marie Curie International Reintegration Grant from the European Union (PIRG-GA-2009-256413), research funds from the Fundació Bosch i Gimpera, and a grant from the Spanish Ministry of Economy and Competitiveness (FFI-2010-20634).

English Linguistics 30: 2 (2013) 463-484 -463-

(C) 2013 by the English Linguistic Society of Japan 
domain just mentioned, or, more likely I think, ${ }^{1}$ the very same one recycled in a variety of cognitive contexts - underlies this ability is by now wellestablished. It is the central lesson of Chomsky (1957), and it captures in a mechanical way Humbolt's insight about "making infinite use of finite means." As Hume (1890: Book III) anticipated, positing such a mechanism ("such a method of proceeding") is "conformable to the usual maxims, by which nature is conducted, where a few principles produce all the variety we observe in the universe, and everything is carry'd on in the easiest and most simple manner."

In the domain of language, this recursive procedure is now standardly called Merge. As should be obvious, no reflection on Merge is alien to biolinguistic considerations. The very act of positing something like Merge is motivated by biolinguistic considerations, understood as an attempt to go beyond the limits of behaviorism and look into the underlying biological substrate of linguistic knowledge. In other words, no Merge without mentalism. But the purpose of this paper is to see if one can go beyond this fundamental biolinguistic conclusion, and shed light on the biological substrate of Merge. Some will find this project pointless, saying that Merge is a biological object already_ "at a suitable level of abstraction," much like theoretical linguistics is often said to be biology "at a suitable level of abstraction" (Boeckx and Piattelli-Palmarini (2005: 462), Berwick (2011b: 461)). But as I have discussed elsewhere (see especially Boeckx (2012)), this characteristic stance of what one could call the first phase of biolinguistics is to be supplanted by a more direct engagement with the biology literature. As Jackendoff (2011: 588) puts it, for biolinguistics to flourish, linguistics ought to "make contact with biology, taking the study of language beyond just the description of languages and language universals." It is in the context of this goal of "interfacing linguistic theory with biology" (Jackendoff (2011: 589)) that this paper must be understood.

Focusing on Merge in this context of renewed attention to biological feasibility/plausibility makes a lot of sense. As Jackendoff (2011: 591) correctly points out, "[t]he minimalist program reconstructs syntactic theory

1 There is already existing material to build on in order to substantiate the claim that Merge is the underlying force behind this various behavioral traits: On Merge and mathematics, see Chomsky (2008), Tutusaus (2012); on Merge and metrics (which I believe may underlie music), see Berwick (2011b), and also Katz and Pesetsky (2009). As for our moral sense, it is likely to build on the detailed event representations (Pietroski (2005), Hurford (2007)) that Merge gives rise to (Boeckx (2009c, To appear)). 
around Merge as the central computational operation." ${ }^{2}$ I would even go further and say that the minimalist program, rigorously pursued, amounts to an attempt to reconstruct linguistic theory (not just syntactic theory) around Merge (see Boeckx (To appear)). Although Merge is not explicitly mentioned in Hauser, Chomsky, and Fitch (2002), it figures prominently in their claim that the Faculty of Language in the Narrow sense (FLN) - that which is specific to humans - likely consists solely of the ability to build syntactic structures recursively and map these to the systems of 'sound/sign' and 'meaning.' It is not hard to recognize Merge in statements like "the core computational mechanisms of recursion as they appear in narrow syntax and the mappings to the interfaces" (p. 1573, column 2-3).

Not surprisingly, many theoretical linguists (see, e.g. Boeckx (2009b), Hornstein (2009)) thought of focusing on Merge when they came across the following 'desiderata' for biolinguistics formulated by biologists:

We need to distill what we know from linguistic theory into a set of computational primitives, and try to link them with models and specific principles of neural computation ...

Thus we need linguistic models that are explicit about the computational primitives (structures and operations) they require, and that attempt to define linguistic problems at a fine enough grain that one can discuss algorithmic and implementational approaches to their solution. We need a list of computations that linguistic theorists deem indispensable to solve their particular problem (e.g., in phonology, syntax, or semantics).

(Fitch (2009: 298))

Linguists and psycholinguists owe a decomposition (or fractionation) of the particular linguistic domain in question (e.g. syntax) into formal operations that are, ideally, elemental and generic. The types of computations one might entertain, for example, include concatenation, comparison, or recursion. Generic formal operations at this level of abstraction can form the basis for more complex linguistic representation and computation.

(Poeppel (2005: 3))

In the next section I will take the reflections in Boeckx (2009b) and Hornstein (2009) as my point of departure, but before doing so, I'd like to highlight one thing that the passage by Fitch just quoted makes clear (Poeppel thinks along similar lines, see Poeppel (2012)): a fruitful biolinguistic discussion of what Merge is will have to combine theoretical con-

${ }^{2}$ Witness the dominant position Merge occupies in Chomsky (2012). 
siderations that are typical of generative grammar (broadly construed), but also considerations at other levels of analysis that are more typical of the writings of David Marr (Marr (1982)) or, in the context of language, of Eric Lenneberg (Lenneberg (1967)). As Lenneberg (1964: 76) wrote 50 years ago, "[n]othing is gained by labeling the propensity for language as biological unless we can use this insight for new research directions - unless more specific correlates can be uncovered." Let this serve as the guideline in the pages that follow. ${ }^{4}$

\section{What Is Merge?}

In the minimalist literature, Merge is taken to be the basic combinatorial operation in syntax (Fukui (2011)). Traditionally (see Chomsky (1995)), it is taken to work as follows: it takes two constituents (minimally, elements selected from the pre-syntactic lexicon) as input and combines them to form a novel constituent labeled by one of the inputs: ${ }^{5}$

(1) $\{\alpha\}+\{\beta\} \rightarrow\{\alpha,\{\alpha, \beta\}\}$

Both Boeckx (2009b) and Hornstein (2009) ask if Merge so conceived can be regarded as an 'atomic,' 'primitive' operation, and put forth the idea that it is an operation complex, consisting of at least two sub-operations: a 'combine' operation and a 'label' operation. ${ }^{6}$ Both suggest that decomposing Merge in this way can be fruitful in the context of figuring out its phylogeny. Merge as a package may be distinctive of humans (and perhaps specific to language), but its sub-operations may be more generic, and shared (in a scattered, mosaic-like fashion) across cognitive domains. While this is quite likely to be the case for the 'combine' part of merge (putting two things together is as basic as it gets), it is less obviously

3 Thanks to Pedro Tiago Martins for reminding me of this statement.

${ }^{4}$ As Paul Pietroski pointed out to me in a related context, taking passages like Lenneberg's seriously amounts to adding another flavor to the 'I-' in 'I-linguistics': in addition to 'individual,' 'internal,' and 'intentional,' our perspective should be 'implementational' (our linguistic constructs should have biological plausibility, they should be biologically implementable).

5 In addition, as summarized by Hornstein (2009: 13), Merge is subject to other conditions, such as the Extension condition (imposing monotonicity), or the Binarity restriction. I set these aside for now.

${ }^{6}$ Perhaps, this is also what Hauser et al. (2002) had in mind when they talked about "the core computational mechanisms of recursion" (note the plural), although I can't be sure, since they don't provide any details. 
true for the 'label' part. In fact, unlike Boeckx (2009b) (who takes labeling to be an instance of generic copying), Hornstein (2009) takes labeling to be sui generis, and goes on to derive central features of natural language grammars from it.

Commenting on Boeckx (2009b), Chomsky (see p. 51 of Piattelli-Palmarini et al. (2009)) agrees to 'decomposing' Merge (as traditionally defined, i.e., as having labeling built into it), but suggests we keep the term 'Merge' to refer to the basic 'combine' operation (Merge tout court), and take 'labeling' to be the result of a Minimal Search algorithm (see Chomsky (2007, 2008, 2013); see also Ott (2012), Narita (2010)). He also suggests that the 'big fact' about Merge is that it is unbounded.

In what follows, I will adopt Chomsky's suggestion and refer to 'unbounded combine' as Merge, defined as follows:

(2) Merge $($ simpliciter $)=_{\text {def }}$ $\alpha \oplus \beta \rightarrow\{\alpha, \beta\}$

And I too want to put real emphasis on the unbounded character of the operation. Extending a line of argument I pursued in Boeckx (2011a, b), I argue that the shift from mere combination to unbounded combination is akin to a phase transition. That is, the relationship between 'combine' and 'Merge' is like that between water and ice. They are and aren't the same thing. ${ }^{7}$ Such a phase transition was made possible because of the nature of the elements manipulated by the operation: whereas concepts impose selectional restrictions (valency requirements) that limit combinations, lexical items don't, or so I claim (see also Pietroski (2007)). Once such selectional constraints were lifted, concepts clothed as lexical items could combine across modular boundaries. ${ }^{8}$

As I have argued elsewhere (Boeckx (2011a, b, 2013)), this claim provides us with a basis to understand the robust results from numerous comparative studies in psychology showing that mature linguistic creatures transcend many cognitive limits seen in animals and prelinguistic infants (infants whose linguistic capacity has not matured yet) (see Spelke (2003), Carruthers $(2003,2006))$. It can also make sense of the species-specific

7 As I argue in Boeckx (2013), this is a more biologically realistic way of thinking about the novel character of the language faculty than the problematic FLN/FLB distinction.

${ }^{8}$ As I discuss in Boeckx (2013), this phase transition can be understood in brain terms, and correspond to the emergence of a globular brain, and its attendant connectivity consequences. See also the discussion in the next section. 
cognitive fluidity reflected in the archeological records (see Mithen (1996)).

I am aware that claiming that lexical items don't impose selectional restrictions, thereby allowing for unbounded combination (Merge proper) departs from standard wisdom and traditional models of grammar (including the latest minimalist views), which take Merge to be constrained by, or, better said, driven by (or structured by) the features of the units it combines. ${ }^{9}$ This traditional view, which I have dubbed 'lexiconcentric' in Boeckx (To appear), is widely shared across frameworks, which take properties of 'words' (rather than merge) to be the key evolutionary innovation (Pinker and Jackendoff (2005), Jackendoff (2011), Bouchard (2013), Koster (2009), even though sometimes they claim that "all you need is merge" (the title of Berwick (2011b)). Consider the following passages, and the conflicting claims they contain: ${ }^{10}$

It is not the lack of words alone that blocks the possibility of more complex birdsong syntax. ...

The key difference is the use of a verb or noun's features to label an entire word sequence with a single label, ...

The selection of a privileged element in this way renders the underlying structure fundamentally asymmetric. ...

This is true precisely because birds apparently do not have words or manipulate word features at all. This is one difference between the human language syntactic system and birdsong.

(Berwick et al. (2012))

I too claim that a property of lexical units was the key, but this property is not valency, but rather, the absence of it (lexical items as 'edge feature carriers,' see Boeckx (2011b, To appear)).

The 'combine' part of (traditional) Merge having been clarified, what of

9 For example, Pesetsky and Torrego (2006) impose a 'vehicle' requirement' on Merge, and Collins (2002) uses lexical features to structure Merge (reconstructing projection, etc.).

${ }^{10}$ In a related publication, Robert Berwick writes that "In particular, the Minimalist Program posits that the human syntactic engine consists of just two components: (1) words and word features; and (2) a single, simple recursive operation, Merge, that glues together words and word complexes into larger units. ... This chapter demonstrates how just these two components, without further stipulation, interact to yield many, perhaps all, of the special design features of human language syntax." (Berwick (2011a: 65)) 
labeling? ${ }^{11}$ I agree with Chomsky (see again p. 51 of Piattelli-Palmarini et al. (2009)) that endocentric labeling is sort of an epiphenomenon (see also Narita (2010)), and should not be forced into syntactic structure building, neither via a clever use of features (Collins (2002)) nor as an operation (be it sui generis or not; see Hornstein (2009), Boeckx (2009b)), but I would like to avoid a mere re-statement of the issue as Chomsky himself (Chomsky (2013)) or Narita (2010) do when they appeal to an H-XP schema (or an even more elaborate version, like the functional sequence of Starke $(2001,2010)$ and Adger (2013)). Instead, I propose we view labeling as the outcome of an interaction between the conceptual repertoire we share with other species that provides general substantive notions that act as proto-labels (to be refined via grammaticalization) and a structural asymmetry that is inherent to syntax: partial, cyclic Spell-Out.

As I discuss in Boeckx (2009a, To appear), the structure of Cyclic SpellOut put forth in Chomsky (2000) and subsequent work combined with Marantz's (2008) suggestion that 'light' (phasal) categories label roots, yield an asymmetric Head-Phrase schema (with the head corresponding to the phase head, and the phrase to the so-called phase complement) that does not require any new syntactic operation (or pre-syntactic lexical information) to generate sets that are, for the external systems interfacing with syntax, more structured than sets generated by bare Merge.

$$
\{\alpha,\{\alpha, \beta\}\}=\{\underbrace{x}_{\text {Phase Head/Cyclic Node Phase Complement/Transferred Material }}, \underbrace{\{\sqrt{\alpha},(\beta)\}}\}
$$

In the publications I referred to already (Boeckx (2009a, To appear)), I observe that an unbounded merge system automatically requires a cyclic chunking operation if it is to be usable at all by the interpretive systems. The reasoning is familiar from the psychology literature on bounded memory and the need for chunking (Miller (1956)). Put differently, phasal spell-out is a generic solution to the problem posed by the emergence of unbounded combination (Merge). The separation cyclic spell-out introduces between what gets transferred and what doesn't (phase edge vs. phase complement in the terminology of Chomsky (2000)) does not recapitulate all the

11 Regarding the conditions standardly imposed on Merge, in particular monotonicity and binarity, I take the former to be a generic 'good design' principle (no rewriting/ reanalysis), whereas the latter falls out from the context in which Merge operates in language (see Boeckx (To appear) for a specific proposal). It is certainly not specific to Merge, as it is absent from it in the context of our number sense (Chomsky (2008)), and maybe also in the context of metrics (Berwick (2011b)). 
(substantive) asymmetries built into labeling in more traditional frameworks, but it does offer a basic formal asymmetry on which to graft notions that arguably belong to the interpretive systems (categories, valuation/licensing schemas, and so on).

The picture that emerges from the considerations in this section is that the structural backbone known as natural language syntax boils down to a merge operation (unbounded combination), made possible by the lifting of valency constrained imposed by concepts (lexical items as edge feature carriers), and made usable (i.e. structured) by a cyclic transfer mechanism (phasal spell out). Syntax, then, essentially takes the form of a structural rhythm, a sequence of merge applications interleaved with spell-out points: Merge steps-Spell-Out, Merge steps-Spell-Out, Merge steps-Spell-Out, .... ${ }^{12}$

As I will argue in the next section, the idea that syntax is a structural rhythm is significant, as it can be used to bridge the mind/brain divide, thanks to the well-studied rhythmic properties of the brain (Buzsáki (2009)). It is also significant because this view of syntax brings it into the fold of a larger class of models inspired by Turing (1952): reaction-diffusion (RD) mechanisms. In the hands of Gierer and Meinhardt (1972) RD systems have been used to account for many important types of pattern formation and morphogenesis observed in development. RD systems consist of a short-range autocatalytic substance, the activator, and of its long-range antagonist, the inhibitor. The inhibitor slows down the production of the activator, as Figure $1^{13}$ illustrates.

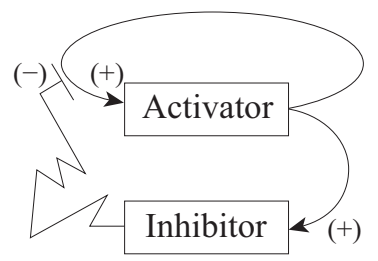

Figure 1: Short-range activator and long-range inhibitor in Gierer-Meinhardt model

It is not hard to see how a natural language system fits into this model:

12 To the best of my knowledge, Richards (2011) was the first to highlight the presence of an alternation of phase heads and non-phase heads in syntax, which I interpret as a structural rhythm.

13 Taken from http://www.scholarpedia.org/article/Gierer-Meinhardt_model 
repeated application of local merge produces the introduction of cyclic nodes that inhibits merge (spell out, or 'de-merge' in the terminology of Fukui (2006)). If this is the right way of looking at things, syntax may be a specific application of a very generic, 'third-factor' mechanism of morphogenesis.

One final point concerning the perspective advocated here. If Merge amounts to what I have claimed here, natural language syntax obeys what Deacon (2005) calls an 'organicist' logic, as opposed to an 'reductionist' logic; an epigenetic logic as opposed to a genetic logic. The present approach indeed conceives of syntactic structuring as embryologists conceive of development: as the gradual, progressive emergence of structural details (parts) out of a generic whole. This is in sharp contrast with the way engineers think of development, with parts being assembled into a whole. I agree with Deacon that an organicist logic is likely to bring us closer to understanding how the brain works, and this is the topic to which I now turn.

\section{How Could Merge Be Implemented in the Brain?}

The claim that a structural rhythm may constitute the backbone of grammar has, in my view, a significant advantage when it comes to figuring out the nature of the 'slash' between mind and brain (mind/brain): it relies on a notion (rhythm) that neuroscientists have already established is central to the workings of the brain. The 'translation' of syntactic structure into rhythmic structure could be just what we need to move from (to use Marrian terminology) the computational level to the algorithmic level, and from there to the implementational level, for rhythms are intrinsic to neuronal activity. It's how neuronal ensembles organize themselves.

Although much of the neuroscience that addresses language-related issues continues to focus on regions and (more recently) pathways connecting these regions, knowing where the brain does things does not tell us what it does, which I take to be the goal of neuro-/bio-linguistics. Isolating regions, of course, could lead to the discovery of cytoarchitectural properties of these regions that may be essential in understanding how exactly the brain does what it does, but this is Marr's implementational level, not the algorithmic level. For the latter, it seems to me that studying the role of rhythms is more fruitful. It strikes me as just the right level of abstraction. Evidence of the fruitfulness of this approach in the language domain comes from the work of David Poeppel, beginning with Poeppel (2003) and culminating with Giraud and Poeppel (2012). 
What Poeppel and colleagues have shown is that by focusing on the endogenous rhythms generated by the cortex, it is possible to understand (as opposed to merely localizing) the cerebral specialization for speech perception and production, and to shed light on the nature of phrasal phonology. The main thesis is that neuronal oscillations contribute to cognition in several ways: for example, by segregating information and organizing spike timing. Specifically, a series of oscillations (in the delta, theta and gamma ranges) appear to be able to track the dynamics of speech. In doing so, they 'chunk' or 'package' incoming information into units of the appropriate temporal granularity. This packaging corresponds to units of phrasal phonology (linking the algorithmic and computational levels). ${ }^{14}$

Going back to syntax, I think that even impressionistically speaking it should be obvious that rhythmic properties may have an explanatory role to play. After all, the central notion of the cycle (see Lasnik (2006) for review), arguably the most innovative concept in generative grammar, is all about rhythm (periodic processing/sampling). In the previous section, I've suggested that constituents arise as a result of the rhythmic organization of Merge and Spell-Out. The particular combination of these two operations gives rise to nested structures (products of merge are embedded inside phases; cf. the notion of 'phase head complement' in Chomsky (2000)). The hypothesis, then, is that syntactic hierarchy reduces to a hierarchical organization of Merge- and Spell-Out-rhythms.

Interestingly, this type of embedding is something that the brain is known to do: numerous studies have demonstrated first that the brain generates a range of oscillations (alpha, beta, gamma, delta, and theta oscillations have figured prominently in the literature), and second that these rhythms interact with one another by influencing (modulating) the phase and/or the amplitude of the oscillations (see Buzsáki (2009) for an excellent overview). Crossfrequency couplings give rise to a hierarchical organization of brain rhythms that underlies many aspects of human cognition. I submit that this element of the "syntax of the brain," as Buzsáki (2010) has called it, provides a

14 Theoretical linguists are not used to thinking of structural representations as the outcome of certain derivational rhythms, not even in phonology, where rhythm may be more obviously central than in syntax. But I believe that Vergnaud (2003) was right to stress the correspondence between the metrical grids familiar to phonologists and the way clocks work, and that this could constitute a first necessary translation step between mind and brain in this domain. 
general ${ }^{15}$ algorithmic substrate, a coding mechanism, for natural language syntax.

It stands to reason that because the mechanisms of syntax put forth in this section are so generic, the specificity of natural language syntax must be found in the anatomical/cytoarchitectural context in which the relevant computations take place. In other words, the relevant specificity must be rooted in the regions involved, in the ways in which they are connected, and in the specific rhythmic frequencies at work.

I have argued in related work (Boeckx (2013)), in line with much recent work (see, e.g. Fedorenko et al. (2010)) that the brain models that biolinguists should entertain should be far more distributed, and less corticocentric, than the classical Broca-Wernicke-Lichtheim-Geschwind model. If I am right that the cognitive signature of our Merge-based syntax is crossmodular concept formation, the 'neuronal workspace' model formulated in Dehaene et al. (1998) in the domain of consciousness (see also Tononi and Edelman (1998)), or the multiple-demand system of Duncan (2010) appear to be of the right kind. The neuronal workspace model emphasizes the role of distributed neurons with long-distance connections, particularly dense in prefrontal, cingulate, and parietal regions, interconnecting multiple specialized processors and "broadcasting" signals at the brain scale in a spontaneous and sudden manner, forming a 'global neuronal workspace.'

In addition to cortical areas, I have argued that the role of the thalamus should not be underestimated. In fact, in the context of the proposal made in this paper, the thalamus appears to be central. Outside of language proper, the thalamus has routinely been assigned a key role in controlling attention, regulating oscillations generated in the cortex, etc. (see Saalmann et al. (2012), Miller and Buschman (2012), among many others). ${ }^{16}$ I'd like

15 'General' here is meant in the sense of 'generic' and 'conserved.' Using the terminology of Chomsky (2005), one could speak of a third-factor effect, the hypothesis here being that natural language syntax runs on neural syntax. Ultimately, then, syntactic constraints reduce to constants of brain oscillations established long before the language faculty emerged. For instance, constraints like Richards's (2010) Distinctness Condition, which prohibits the presence of more than one lexical unit of the same category within the phase complement, may result from constraints imposed by how many distinct rhythms the brain can couple in particular activities. Harmonic constraints like the Finalover-Final Constraint (Biberauer et al. (2008)) may also be the product of constraints imposed by the syntax of the brain, interacting with more specific factors (e.g. languagespecific stress conditions).

16 Buzsáki (1991) goes so far as calling the thalamus a 'clock.' 
to suggest that in the case of language, the thalamus also acts as an oscillation regulator (a reference 'clock'), acting as a relay between activity in the frontal lobe and in the parietal lobe. More precisely, I claim that the thalamus acts as a modulator of oscillatory synchrony, regulating network interactions, and shaping the communication between various brain areas.

Given that the thalamus projects widely, it is in an ideal position to regulate activity across cortical regions. The characteristic, low frequencies it generates may provide just the sort of 'carrier wave' on which higher frequency oscillations across the cortex (typically in the gamma range) can become entrained. (More than merely relaying information, the thalamus, by generating slow rhythms, provides the key 'embedding' step). The recruitment of the thalamus in the context of language would, then, have been required to achieve the nesting of high frequency oscillations inside lower frequency oscillations, much like the more sparsely distributed (less frequent) phase heads were required to embed the (frequent) instances of merge, organizing these into constituents. Put another way, thalamic activity would correspond to a periodic sampling of cortical (fronto-parietal) activity ("cyclic spell-out"). Once synchronized, the fronto-pariental network would then act on other specific regions (including Broca's area, but also subcortical structures) to arrive at more detailed linguistic ${ }^{17}$ representations (including, ultimately, linear order; see Boeckx and Martínez-Álvarez (2013)), following the organicist logic mentioned in the previous section.

Needless to say, many details at the implementational level (e.g. the characterization of the specific low frequency oscillation generated by the thalamus) remain to be determined by experimental work, but I hope that the outline of the model is clear, at least. As far as the cortex is concerned, I am betting on a fronto-parietal network for several reasons. First, recent work trying to match syntactic complexity onto the brain has isolated this network (see especially Ohta, Fukui, and Sakai (2013)). Second, if Ben Shalom and Poeppel (2008) are right in assigning to the parietal region a general "analyzing" function, and to the frontal region an equally general "synthesizing" function, the fronto-patietal ensemble may provide just the right substrate for an 'Analysis-by-Synthesis' approach of the sort that ap-

17 As Boeckx and Martínez-Álvarez (2013) point out, these regions may also be crucial in non-linguistic tasks, e.g. the hierarchical processing tasks that are part of Action Grammar models, which, as Fujita (2009) pointed out, bear a family resemblance with linguistic representations. 
pears well-motivated for language and cognition more generally (see Bever and Poeppel (2010)). And third, a rich fronto-parietal network is the outcome of the species-specific globular character of sapiens' brain (a "neomorphic hypertrophy of the parietal volumes, leading to a dorsal growth and ventral flexion (convolution) and consequent globularity of the whole structure" (Bruner (2004)). It therefore makes sense to ascribe to it a speciesspecific trait like natural language syntax. ${ }^{18}$

The sapiens-specific globularization of the brain, then, may have set the anatomical stage for the phase transition that I have called Merge. Of course, other species, in particular our extinct cousins the Neandertals, had big brains, and thalamo-fronto-parietal connections, but perhaps not of the right balance to achieve the phase transition that provided the basis for language and our distinct cognitive profile (cross-modular concept formation). This is perhaps due to the fact that a significant portion of their brains had to be devoted to other considerations (see Pearce, Stringer, and Dunbar (2013) for a specific suggestion involving differences in visual processing). At the moment, we don't know enough to be sure, but it is interesting to note that one of the genes involved in the shape of the cranium, and whose expression differs in us and in Neandertals (Meyer et al. (2012)) has recently been shown to be under the regulation of clock-genes (Reale et al. (2013)). The fact that RUNX2 is expressed in the thalamus may reinforce the position defended in this section. ${ }^{19}$ But this awaits further research.

\section{Implications}

The central claim of this paper is that in order to reflect on the brain basis (and from there onto the molecular basis) of syntax, the core operation called Merge should be formulated in as much a generic way as possible. Perhaps the most important step in achieving this is to free syntactic combination from lexical influence, or, to put it in another way, to abandon the

18 See also Mantini et al. (2013) for recent evidence in favor of a richer fronto-parietal network in the context of primate evolution.

${ }^{19}$ It is not inconceivable that the rhythmic expression of RUNX2 may provide a molecular basis for its clock function in the brain, much like the 'Clock-Wavefront' model of somitogenesis put forth by Cooke and Zeeman (1976) (conceptually related to reactiondiffusion models) was shown to be implemented via the rhythmic expression of genes under the control of Wnt and Notch signaling Pourquié (2003). Nature really does seem to go settle on a few implementational paths, and molecularly implements 'cycles' in a unique fashion to achieve segmentation/chunking of all kinds. 
idea that syntax begins with already structured 'word-like' elements.

I have argued here, building on previous work (beginning with Boeckx (2009a)), that syntactic computation boils down to a rhythm: an interleaving of Merge and Spell Out applications. This has allowed me to suggest that this mind-level description could be put in correspondence with brain-level descriptions that assign a crucial role to rhythms. I have also argued that this type of computation requires an appreciation of the role of sub-cortical structures, in particular the thalamus.

If this is correct, it should be quite clear that the methodology pursued to bring us closer to the syntax of the brain takes us ever further away from the syntax of specific languages. This I view as the sharpest expression of the difference between (rigorous or radical) syntactocentrism and lexicocentrism, or indeed the dividing line between (traditional, even if modern) theoretical linguistics and biolinguistics. ${ }^{20}$ The commonsensical approach of starting with word-like things may be right to produce grammars (even generative grammars), but wrong to characterize the faculty that underlies grammar development. As a matter of fact, this is a lesson that should not surprise those who are familiar with the language acquisition literature. Although it seems that children first acquire words, and then combine them into sentences, word learning requires syntactic bootstrapping (see Gleitman et al. (2005)). ${ }^{21}$

Naturally, the primacy of syntax has implications for how grammars (Ilanguages) are attained: if lexical structure is not given in all its richness

20 This point has been well expressed in Hall (2012), where he asks "What does it mean to pursue a biolinguistic approach to phonology, or to language in general?" Currie Hall is right in saying that

In one sense, it means that the field of inquiry becomes broader. Some phonological patterns find an explanation in the interface conditions of syntax, others in the neurophysiological underpinnings of mammalian auditory perception. A successful biophonologist must know enough about both of these things, and many others, to be able to make reasonable inferences about what each of them contributes. ... In another sense, the central object of study becomes much smaller. If some of phonology is really syntax, and some of it is really phonetics, then relatively little of phonology is phonology.

This is where, I think, biolinguistics meets (some kind of) minimalism, and it is also where biolinguistics really departs from philology-oriented projects.

21 Equivalently, prosodic bootstrapping, which I think amounts to the same thing - an option not considered Millotte et al. (2013) in their otherwise excellent review-, if prosody - a rhythm! - is indeed a very faithful reflex of syntactic constituency, as argued in Samuels (2011) and references therein. 
and complexity in the pre-syntactic lexicon, it must come from somewhere else. Whereas I think that syntagmatic grammar (syntax, essentially) is a particular expression of the syntax of the brain, it seems to me that paradigmatic grammar (the 'lexicon,' the Construction Grammar, morphophonology) requires us to take seriously the structuring role of socio-cultural transmission and learning, as many scholars have suggested (the works of Simon Kirby or Luc Steels come to mind here, as does Blevins (2004); see also Boeckx et al. (In press)). Put differently, to understand the nature of Ilanguages completely, biolinguistics must join forces with sociolinguistics. ${ }^{22}$

A particular consequence of this in the context of language evolution is that there may have been a (possibly substantial) gap between the emergence of natural syntax (the backbone of our species' language of thought) and the emergence of I-languages in all their complexity, which may have had to wait for the right context of transmission to arise. Put another way, there may well have been a stage of 'syntactic protolanguage' preceding the rise of full-blown languages. Put yet another way, if the mind is what the brain does, humans were cognitively modern when they became anatomically modern, but they may have had to wait to construct their niche and become behaviorally modern.

The last point I'd like to make in this concluding section concerns a question raised by Moro (2008: 179). Moro asks why, if language and more specifically its core syntactic engine is ultimately a fact about genetics, why don't we find human beings lacking the ability to develop a syntactic competence and pass this 'genetic disorder' to their offsprings. It seems to me that this question assumes that innateness entails genetic determinism. But this is not so: the development of an Extended Synthesis (Pigliucci and Müller (2010)) in biology makes it clear that the options of non-genetic nativism are vast. In addition, if the present take on the brain basis of Merge is correct, syntactic competence is an immediate product of an anatomically modern human brain. You can't lack one without lacking the other.

Having said this, the proposal I have put forth does make predictions regarding certain linguistic disorders. It predicts that many grammatical disorders amount to disruptions of the normal syntactic rhythm. That is, many such disorders should be viewed as "rhythmopathiesm," "oscillopathies" or "dysrhythmias." In this context, it is interesting to note that patients with

22 This complementarity does not, however, cast doubt on the biolinguistic program, as Balari et al. (2012) argue in their reply to claims like Koster (2010) that it does. 
bilateral paramedian thalamic infarction have been reported to exhibit severe syntactic deficits ("characterized in our patient by simple sentences and sentence fragments with a complete absence of embedded clauses" (De Witte et al. (2006))). It is also important to realize that because of the crucial 'interfacing' role syntax has, there won't be any pure 'syntactic' deficit. It will always be accompanied by deficits in the interpretive systems. In the context of his reflection on syntactic deficits, Moro (2008: 180) writes that "[d]ata in the literature suggest syntactic damage, but it is never completely independent of the semantic component," and for this reason puts such data aside, but this is in fact exactly what one should expect, especially when it comes to the syntax-semantics interface. As Hinzen $(2006,2007)$ has put it, there is no semantic component independently of syntax. If Pietroski (To appear) is correct in defining linguistic meanings as "mechanically executable instructions (or recipes) for how to build systematically composable concepts that are monadic and conjunctive," such meanings are syntactic instructions. There really is no escape from syntax.

\section{REFERENCES}

Adger, David (2013) The Syntax of Substance, MIT Press, Cambridge, MA.

Balari, Sergio, Cedric Boeckx and Guillermo Lorenzo (2012) "On the Feasibility of Biolinguistics: Koster's Word-Based Challenge and Our 'Natural Computation' Alternative," Biolinguistics 6, 205-221.

Ben Shalom, Dorit and David Poeppel (2008) "Functional Anatomic Models of Language: Assembling the Pieces," The Neuroscientist 14, 119-127.

Berwick, Robert (2011a) "Syntax Facit Saltum Redux: Biolinguistics and the Leap to Syntax," The Biolinguistic Enterprise: New Perspectives on the Evolution and Nature of the Human Language Faculty, ed. by Anna Maria Di Sciullo and Cedric Boeckx, 65-99, Oxford University Press, Oxford.

Berwick, Robert (2011b) "All You Need Is Merge: Biology, Computation and Language from the Bottom-Up," The Biolinguistic Enterprise: New Perspectives on the Evolution and Nature of the Human Language Faculty, ed. by Anna Maria Di Sciullo and Cedric Boeckx, 461-491, Oxford University Press, Oxford.

Berwick, Rorbert, Gabriel Beckers, Kazuo Okanoya and Johan Bolhuis (2012) "A Bird's Eye View of Human Language Evolution," Frontiers in Evolutionary Neuroscience 4, Article 5, 1-25.

Bever, Tom G. and David Poeppel (2010) “Analysis by Synthesis: A (Re-)Emerging Program of Research for Language and Vision," Biolinguistics 4, 174-200.

Biberauer, Teresa, Anders Holmberg and Ian G. Roberts (2008) "Structure and Linearization in Disharmonic Word Orders," WCCFL 26, 96-104. 
Blevins, Juliette (2004) Evolutionary Phonology: The Emergence of Sound Patterns, Cambridge University Press, Cambridge.

Boeckx, Cedric (2009a) "The Locus of Asymmetry in UG," Catalan Journal of Linguistics 8, 41-53.

Boeckx, Cedric (2009b) "On the Nature of Merge," Of Minds and Language: A Basque Encounter with Noam Chomsky, ed. by Massimo Piattelli-Palmarini, Pelloo Salaburu and Juan Uriagereka, 44-57, Oxford University Press, Oxford.

Boeckx, Cedric (2009c) "Some Notes on the Syntax-Thought Interface," Proceedings of the Sophia University Linguistic Society 24, 92-103, Sophia University Linguistic Society.

Boeckx, Cedric (2011a) "The Emergence of Language, from a Biolinguistic Point of View," The Oxford Handbook of Language Evolution, ed. by Maggie Tallerman and Kathleen Gibson, 492-501, Oxford University Press, Oxford.

Boeckx, Cedric (2011b) "Some Reflections on Darwin's Problem in the Context of Cartesian Biolinguistics," The Biolinguistic Enterprise: New Perspectives on the Evolution and Nature of the Human Language Faculty, ed. by Anna Maria Di Sciullo and Cedric Boeckx, 42-64. Oxford University Press, Oxford.

Boeckx, Cedric (2012) "The I-Language Mosaic," Language from a Biological Point of View: Current Issues in Biolinguistics, ed. by Cedric Boeckx, Mamen Horno and Jose-Luis Mendivil, 23-51, Cambridge Scholars Publishing, Newcastleupon-Tyne.

Boeckx, Cedric (2013) "Biolinguistics: Forays into Human Cognitive Biology," Journal of Anthropological Sciences 91, 1-28.

Boeckx, Cedric (To appear) Elementary Syntactic Structures, Cambridge University Press, Cambridge.

Boeckx, Cedric, Evelina Leivada and Pedro Tiago Martins (In press) "Language and Complexity Considerations: A Biolinguistics Perspective," Llengua, Societat $i$ Comunicació.

Boeckx, Cedric and Anna Martínez-Álvarez (2013) “A Multi-Step Algorithm for Serial Order: Converging Evidence from Linguistics and Neuroscience," paper presented at GLOW 36, Lund.

Boeckx, Cedric and Massimo Piattelli-Palmarini (2005) "Language as a Natural Object: Linguistics as a Natural Science," The Linguistic Review 22, 467-471.

Bouchard, Denis (2013) Nature and Origin of Language, Oxford University Press, Oxford.

Bruner, Emilano (2004) "Geometric Morphometrics and Paleoneurology: Brain Shape Evolution in the Genus Homo," Journal of Human Evolution 47, 279-303.

Buzsáki, Gyorgi (1991) “The Thalamic Clock: Emergent Network Properties," Neuroscience 41, 351-364.

Buzsáki, Gyorgi (2009) Rhythms of the Brain, Oxford University Press, Oxford.

Buzsáki, Gyorgi (2010) "Neural Syntax: Cell Assemblies, Synapsembles, and Readers," Neuron 68, 362-385.

Carruthers, Peter (2003) "The Cognitive Functions of Language," Behavioral and Brain Sciences 25, 657-674. 
Carruthers, Peter (2006) The Architecture of the Mind, Oxford University Press, Oxford.

Chomsky, Noam (1957) Syntactic Structures, Mouton, The Hague.

Chomsky, Noam (1995) The Minimalist Program, MIT Press, Cambridge, MA.

Chomsky, Noam (2000) "Minimalist Inquiries: The Framework," Step by Step: Essays on Minimalist Syntax in Honor of Howard Lasnik, ed. by Roger Martin, David Michaels and Juan Uriagereka, 89-155, MIT Press, Cambridge, MA.

Chomsky, Noam (2005) "Three Factors in the Language Design," Linguistic Inquiry $36,1-22$.

Chomsky, Noam (2007) "Approaching UG from Below," Interfaces + Recursion = Language?: Chomsky's Minimalism and the View from Semantics, ed. by Uli Sauerland and Hans-Martin Gärtner, 1-30, Mouton de Gruyter, Berlin.

Chomsky, Noam (2008) "On Phases," Foundational Issues in Linguistics, ed. by Carlos Otero, Robert Freidin and Maria-Luisa Zubizarreta, 133-166, MIT Press, Cambridge, MA.

Chomsky, Noam (2012) "Introduction," Foundations of Biolinguistics: Selected Writings by Noam Chomsky, ed. by Naoki Fukui, 17-26, Iwanami, Tokyo.

Chomsky, Noam (2013) "Problems of Projection," Lingua 130, 33-49.

Collins, Chris (2002) "Eliminating Labels," Derivation and Explanation in the Minimalist Program, ed. by Samuel D. Epstein and T. Daniel Seely, 42-64, Blackwell, Oxford.

Cooke, J. and E. C. Zeeman. (1976) "A Clock and Wavefront Model for Control of the Number of Repeated Structures during Animal Morphogenesis," Journal of Theoretical Biology 58, 455-476.

De Witte, Lieve, Ineke Wilssens, Sebastiaan Engelborghs, Peter P. De Deyn and Peter Mariën (2006) "Impairment of Syntax and Lexical Semantics in a Patient with Bilateral Paramedian Thalamic Infarction," Brain and Language 96, 69-77.

Deacon, Terrence W. (2005) "Language as an Emergent Function: Some Radical Neurological and Evolutionary Implications," THEORIA: An International Journal for Theory, History and Foundations of Science 20, 269-286.

Dehaene, Stanislas, Michel Kerszberg and Jean-Pierre Changeux (1998) "A Neuronal Model of a Global Workspace in Effortful Cognitive Tasks," Proceedings of the National Academy of Sciences 95, 14529-14534.

Duncan, John (2010) "The Multiple-Demand (MD) System of the Primate Brain: Mental Programs for Intelligent Behavior," Trends in Cognitive Sciences 14, 172-179.

Fedorenko, Evelina, Po-Jang Hsieh, Alfonso Nieto-Castañón, Susan Whitfield-Gabrieli and Nancy Kanwisher (2010) "New Method for fMRI Investigations of Language: Defining ROIs Functionally in Individual Subjects," Journal of Neurophysiology 104, 1177-1194.

Fitch, W. Tecumseh (2009) "Prolegomena to a Future Science of Biolinguistics," Biolinguistics 3, 283-320.

Fujita, Koji (2009) "A Prospect for Evolutionary Adequacy: Merge and the Evolution and Development of Human Language," Biolinguistics 3, 128-153. 
Fukui, Naoki (2006) Theoretical Comparative Syntax, Routledge, London.

Fukui, Naoki (2011) "Merge and Bare Phrase Structure," The Oxford Handbook of Linguistic Minimalism, ed. by Cedric Boeckx, 73-95, Oxford University Press, Oxford.

Gierer, Alfred and Hans Meinhardt (1972) “A Theory of Biological Pattern Formation," Biological Cybernetics 12, 30-39.

Giraud, Anne-Lise and David Poeppel (2012) "Cortical Oscillations and Speech Processing: Emerging Computational Principles and Operations," Nature Neuroscience 15, 511-517.

Gleitman, Lila, Kimberly Cassidy, Rebecca Nappa, Anna Papafragou and John C. Trueswell (2005) "Hard Words," Language Learning and Development 1, 23-64.

Hall, Daniel Currie (2012) "Review Article: Phonological Architecture: A Biolinguistic Perspective (Oxford Studies in Biolinguistics 2) by Bridget D. Samuels, Oxford University Press, Oxford, 2011," Journal of Linguistics 48, 736-741.

Hauser, Marc D., Noam Chomsky and W. Tecumseh Fitch (2002) "The Faculty of Language: What Is It, Who Has It, and How Did It Evolve?" Science 298, 1569-1579.

Hinzen, Wolfram (2006) Mind Design and Minimal Syntax, Oxford University Press, Oxford.

Hinzen, Wolfram (2007) An Essay on Naming and Truth, Oxford University Press, Oxford.

Hornstein, Norbert (2009) A Theory of Syntax, Cambridge University Press, Cambridge.

Hume, David (1890) A Treatise of Human Nature: Being an Attempt to Introduce the Experimental Method of Reasoning into Moral Subjects; and, Dialogues concerning Natural Religion, Longmans, Green, and Company, London.

Hurford, James R. (2007) The Origins of Meaning, Oxford University Press, Oxford.

Jackendoff, Ray (2011) "What Is the Human Language Faculty?: Two Views," Language 87, 586-624.

Katz, Jonah and David Pesetsky (2009) "The Identity Thesis for Language and Music," ms., MIT.

Koster, Jan (2009) "Ceaseless, Unpredictable Creativity: Language as Technology," Biolinguistics 3, 61-92.

Koster, Jan (2010) "Language and Tools," ms., Universiteit Groningen.

Lasnik, Howard (2006) "Conceptions of the Cycle," Wh-movement: Moving On, ed. by Lisa Cheng and Norbert Corver, 197-216, MIT Press, Cambridge, MA.

Lenneberg, Eric H. (1964) “A Biological Perspective of Language," New Directions in the Study of Language, ed. by Eric H. Lenneberg, 65-88, MIT Press, Cambridge, MA.

Lenneberg, Eric H. (1967) Biological Foundations of Language, Wiley, New York.

Mantini, Dante, Maurizio Corbetta, Gian Luca Romani, Guy A. Orban and Wim Vanduffel (2013) "Evolutionarily Novel Functional Networks in the Human Brain?" The Journal of Neuroscience 33, 3259-3275. 
Marantz, Alec (2008) "Words and Phases," Phases in the Theory of Grammar, ed. by Sook-Hee Choe, 191-222, Dong In, Seoul.

Marr, David (1982) Vision, Freeman, San Fransisco.

Meyer, Matthias et al. (2012) "A High-Coverage Genome Sequence from an Archaic Denisovan Individual," Science 338, 222-226.

Miller, Earl K. and Timothy J. Buschman (2012) "Cortical Circuits for the Control of Attention," Current Opinion in Neurobiology 23, 1-7.

Miller, George A. (1956) "The Magic Number Seven, Plus or Minus Two," Psychological Review 63, 81-97.

Millotte, Severine, Elodie Cauvet, Perrine Brusini and Anne Christophe (2013) "Discovering Word Forms and Word Meanings: The Role of Phrasal Prosody and Function Words," The Cambridge Handbook of Biolinguistics, ed. by Cedric Boeckx and Kleanthes K. Grohmann, 86-93, Cambridge University Press, Cambridge.

Mithen, Steven J. (1996) The Prehistory of the Mind, Thames and Hudson, London.

Moro, Andrea (2008) The Boundaries of Babel: The Brain and the Enigma of Impossible Languages, MIT Press, Cambridge, MA.

Narita, Hiroki (2010) Phasing in Full Interpretation, Doctoral dissertation, Harvard University.

Ohta, Shinri, Naoki Fukui and Kuniyoshi Sakai (2013) "Syntactic Computation in the Human Brain: The Degree of Merger as a Key Factor," PLoS One 8, e56230.

Ott, Dennis (2012) Local Instability: Split Topicalization and Quantifier Float in German, de Gruyter, Berlin/New York.

Pearce, Eiluned, Chris Stringer and Robin I. M. Dunbar (2013) "New Insights into Differences in Brain Organization between Neanderthals and Anatomically Modern Humans," Proceedings of the Royal Society of London, Series B, Biological Sciences 280, 20130168.

Pesetsky, David and Esther Torrego (2006) "Probes, Goals and Syntactic Categories," Proceedings of the 7th Annual Conference on Psycholinguistics, ed. by Yukio Otsu, 24-60, Hituzi Syobo, Tokyo.

Piattelli-Palmarini, Massimo, Pello Salaburu and Juan Uriagereka, ed. (2009) Of Minds and Language: A Basque Encounter with Noam Chomsky, Oxford University Press, Oxford.

Pietroski, Paul M. (2005) Events and Semantic Architecture, Oxford University Press, Oxford.

Pietroski, Paul M. (2007) "Systematicity via Monadicity," Croatian Journal of Philosophy 7, 343-374.

Pietroski, Paul M. (To appear) Conjoining Meanings: Semantics without Truth Values, Oxford University Press, Oxford.

Pigliucci, Massimo and Gerd Müller, ed. (2010) Evolution: The Extended Synthesis, MIT Press, Cambridge, MA.

Pinker, Steven and Ray Jackendoff (2005) "The Faculty of Language: What's Special about It?" Cognition 95, 201-236. 
Poeppel, David (2003) "The Analysis of Speech in Different Temporal Integration Windows: Cerebral Lateralization as 'Asymmetric Sampling in Time'," Speech Communication 41, 245-255.

Poeppel, David (2005) "The Interdisciplinary Study of Language and Its Challenges," Technical report, Jahrbuch des Wissenschaftskollegs zu Berlin.

Poeppel, David (2012) "The Maps Problem and the Mapping Problem: Two Challenges for a Cognitive Neuroscience of Speech and Language," Cognitive Neuropsychology 29, 34-55.

Pourquié, Olivier (2003) "The Segmentation Clock: Converting Embryonic Time into Spatial Pattern," Science Signaling 301, 328.

Reale, Meghan E., Ian C. Webb, Xu Wang, Ricardo M. Baltazar, Lique M. Coolen and Michael N. Lehman (2013) "The Transcription Factor Runx2 Is under Circadian Control in the Suprachiasmatic Nucleus and Functions in the Control of Rhythmic Behavior," PLoS One 8, e54317.

Richards, Marc (2011) “Deriving the Edge: What's in a Phase?" Syntax 14, 74-95.

Richards, Norvin (2010) Uttering Trees, MIT Press, Cambridge, MA.

Saalmann, Yuri B., Mark A. Pinsk, Liang Wang, Xin Li and Sabine Kastner (2012) "The Pulvinar Regulates Information Transmission between Cortical Areas Based on Attention Demands," Science 337, 753-756.

Samuels, Bridget (2011) Phonological Architecture: A Biolinguistic Perspective, Oxford University Press, Oxford.

Spelke, Elizabeth (2003) "What Makes Us Smart?: Core Knowledge and Natural Language," Language and Mind: Advances in the Study of Language and Thought, ed. by Dedre Gentner and Susan Goldin-Meadow, 277-311, MIT Press, Cambridge, MA.

Starke, Michal (2001) Move Dissolves into Merge, Doctoral dissertation, Université de Genève.

Starke, Michal (2010) "Nanosyntax: A Short Primer to a New Approach to Language," Nordlyd 36.

Tononi, Giulio and Gerald M. Edelman (1998) "Consciousness and Complexity," Science 282, 1846-1851.

Turing, Alan M. (1952) "The Chemical Basis of Morphogenesis," Philosophical Transactions of the Royal Society of London, Series B, 237, 37-72.

Tutusaus, Jeroni (2012) The Language Byproduct Conjecture and the Formal Definition of Merge: Some Logical Consequences, MA Thesis, Universitat de Barcelona.

Vergnaud, Jean-Roger (2003) “On a Certain Notion of 'Occurrence': The Source of Metrical Structure, and of Much More," Living on the Edge: 28 Papers in Honor of Johathan Kaye, ed. by Steven Ploch, 599-632, Mouton de Gruyter, Berlin/New York.

[received March 24, 2013, revised and accepted July 13, 2013] 
Catalan Institute for Advanced Studies and Research (ICREA) \& Department of Linguistics

Universitat de Barcelona

Gran Via de les Corts Catalanes, 585

08007 Barcelona

Spain

e-mail: cedric.boeckx@ub.edu 